\title{
LA ENFERMERÍA EN LA CORTE REAL ENTRE LA EDAD MEDIA Y LA EDAD MODERNA: ACTIVIDAD ASISTENCIAL EN LA CORTE DE ENRIQUE IV E ISABEL DE VALOIS
} Crespo Muñoz FJ' ${ }^{1}$, Luque Sánchez $E^{2}$, Cañadas de la Fuente GA $^{3}$, González García A ${ }^{4}$.

${ }^{1}$ Licenciado en Historia. Archivo General de Simancas. ${ }^{2}$ Licenciada en Historia. Archivo General de Simancas. ${ }^{3}$ Licenciado en Antropología Social y Cultural. Universidad de Granada. ${ }^{4}$ Licenciado en Antropología Social y Cultural. H.U.

Virgen de las Nieves.

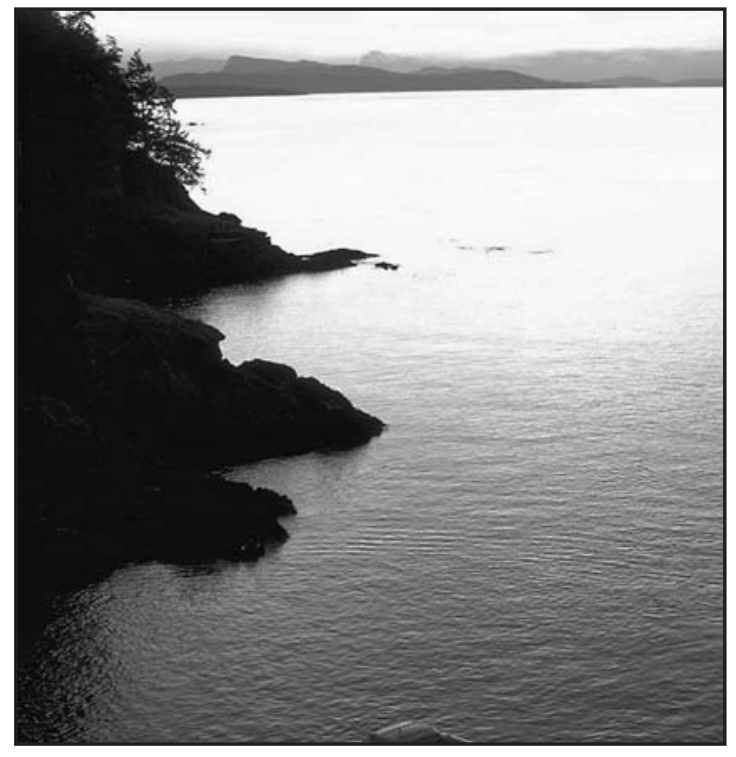

NURSING IN THE ROYAL CHAMBER BETWEEN THE MIDDLE AGE AND THE MODERN AGE: HEALTHCARE IN THE COURT OF ENRIQUE IV AND ISABEL OF VALOIS

\section{ABSTRACT}

$\mathrm{H}$ ealthcare in the court was something fundamental. The different health professionals had as their objective to watch over the health of everybody who belonged to it. Something that everyone of them carried out depending on their abilities and their knowlegde.

The work that we propose is based on the information found in the section of Casas y Sitios Reales of the Archivo General de Simancas.
There, we could find information about the services provided to Enrique IV and Isabel of Valois during their respective reigns, among other monarchs.

Once we had the information analyzed we could confirm the objectivity of a methodical work when it came to provide the care needed, as well as the application of treatments by the health workers, more specifically, nurses.

Key words: History, Nursing, Pharmacy, Archive, Court.

A ENFERMAGEM NA CORTE REAL ENTRE A ICADE MÉDIA E A IDADE MODERNA: ATIVIDADE ASSISTENCIAL NA CORTE DE ENRIQUE IV E ISABEL DE VALOIS

\section{RESUMO}

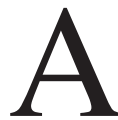

assistência sanitária na Corte Real era algo primordial. Os diversos profissionais de saúde tinham como objetivo zelar pela saúde das pessoas pertencentes à Corte, como uma tarefa que levava a sério cada um daqueles profissionais, dentro de suas competências e do saber próprio de seu cargo.

Para isto, o presente trabalho se baseia na documentação pertencente à seção de Casas e Sítios Reais do Arquivo Geral de Simancas, em que foi possível levantar dados, entre outros, sobre a atenção à saúde prestada por Enrique IV e Isabel de Valois, durante o reinado de ambos. 
Analisada a referida documentação, confirmase a objetividade de um trabalho metódico no momento de se prestar os cuidados e na aplicação de tratamentos pelos profissionais de saúde, mais especificamente, da Enfermagem.

Palavras Chave: História, Enfermagem, Botica, Arquivo, Corte Real.

\section{RESUMEN}

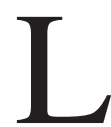

a asistencia sanitaria en la Corte Real era algo primordial. Los distintos profesionales sanitarios tenían como objetivo velar por la salud de las personas pertenecientes a ésta, tarea que llevaban a cabo cada uno de ellos dentro de sus competencias y el saber propios de su cargo.

Para ello, el trabajo que se desarrolla a continuación se basa en la documentación perteneciente a la sección de Casas y Sitios Reales del Archivo General de Simancas, en la que es posible encontrar datos, entre otros monarcas, sobre la atención prestada a Enrique IV y a Isabel de Valois, durante el reinado de ambos.

Analizada dicha documentación, se confirma la objetividad de un trabajo metódico a la hora de prestar los cuidados y la aplicación de tratamientos por el personal sanitario, concretamente del de Enfermería.

Palabras claves: Historia, Enfermería, Botica, Archivo, Corte Real.

\section{INTRODUCCIÓN}

No cabe duda de que la enfermería, como enseñanza reglada, se remonta a siglos pasados, al igual que otras ciencias afines al ámbito sanitario (García Martínez, 1999; Eseverri, 1997), y como disciplina informal, muchísimo más aun (De los Santos, 1999). La presencia de la enfermería y otras figuras sanitarias han ocupado un lugar distinguido en puestos importantes dentro del ámbito social y sus distintos estratos a lo largo del tiempo. Uno de los más destacados y objeto de nuestro estudio es de los responsables de los cuidados de salud en el entorno regio (Campos Díez, 2002).

La Medicina del medievo se basaba, en su vertiente erudita, en las sabidurías hipocráticas y galé- nicas transmitidas a través del mundo árabe. Se puede considerar que el ámbito cortesano sería el más afectado por una ciencia médica tendente a la racionalidad.

Por tanto, la Corte Real, foco principal de nuestra investigación y de la que se tratarán los diferentes aspectos inherentes al trabajo que suponía ser sanitario de la misma, constituye un escenario en el que se debe velar por la salud tanto de la familia real como de los cortesanos, eso sí, con la distinción que su posición en el escalafón social (Cañadas, Luque \& Crespo, 2007); este punto es algo obvio, ya que la relación que unía a ambos era, en la mayoría de casos, bastante más que estrecha y por tanto la coexistencia se daba tanto en estados de salud como de enfermedad. El medicamento fue el medio de encuentro de varios oficios adscritos a la Corte y, por tanto, de una actividad medico-asistencial.

Nuestros objetivos pretenderán determinar, a un nivel básico, la organización de los cuidados de salud en el entorno mencionado anteriormente. Así mismo, profundizaremos en la vida cotidiana del personal sanitario y en las dificultades que se les presentaban en el trabajo y en la forma de desarrollar el mismo.

\section{MATERIAL Y MÉTODOS}

El proceso de investigación se llevo a cabo mediante una revisión bibliográfica y documental en el Archivo General de Simancas, gracias a la cual se aportan una serie de datos que contribuyen notablemente a la historia de la Enfermería en España, más concretamente, durante el periodo comprendido entre los siglos XV-XVI.

La metodología para la realización del trabajo es de tipo cualitativa. La documentación detalla con bastante precisión el quehacer de los profesionales sanitarios de la Corte Real, lo que nos permite ubicarnos en el entorno sociolaboral (De la Cuesta, 2006) y en la percepción que éstos tenían.

Los materiales empleados para el desarrollo de este trabajo abarcan un compendio documental de la sección Casas y Sitios Reales, conservada en el mencionado archivo, relativa a Enrique IV y a Isabel de Valois, entre otros monarcas.

Una vez localizado el material, se comenzó transcribiendo la documentación pertinente a nues- 
tra investigación y, posteriormente, se procedió a realizar un análisis comparativo de su contenido, a fin de llegar a las conclusiones manifestadas (Cañadas, Crespo \& Luque, 2003).

\section{RESULTADOS}

El análisis de la documentación seleccionada implica una ardua tarea, pues la cantidad de personal que había en la Corte Real podía llegar a ser desmesurada.

De entre la información seleccionada a la hora de estudiar los documentos concernientes al personal sanitario que trabajaba en la corte, encontramos datos de gran interés en la parte que hace referencia al material constitutivo de la enfermería, a los tratamientos realizados, al personal que trabajaba con los que eran susceptibles de cuidados para la salud y sobres cuestiones de carácter socioeconómico y administrativo.

En lo que se refiere a las funciones propiamente dichas del personal sanitario, en la documentación relativa a Enrique IV, se pueden apreciar labores propias de la Enfermería. La administración de medicamentos y remedios terapéuticos tales como: "purgaçiones", "lauatorio", "coçimientos", "safumario", entre otras funciones, corrían a cargo de dicho personal (Chocarro, Melgar \& Pérez, 1996; Eseverri, 1996). Se podría deducir que todo ello iría encaminado a la adopción de una metodología científica (Luque, Cañadas, Crespo \& Cantero, 2006) y así sería descrito con posterioridad en la obra de Andrés Fernández, referencia indiscutible para la profesión (Fernández, 1617).

Dentro de la documentación se encontraron los tratamientos que se aplicaban de forma detallada, tal y como se mencionó anteriormente, y, además, la composición y las funciones que tenían cada uno. Así mismo, estaban inventariadas varias relaciones de elementos indispensables para la enfermería, juntos a los cuales se encontraba su tasación económica, debido a que figuraban como los gastos necesarios en un periodo determinado. El material empleado por el personal de enfermería estaba compuesto, entre otras cosas, por un "arca de la enfermería", orinales, vasos para jarabes, ollas y cántaros para hervir agua, cestas, redomas para agua, un "candelero de açófar"; etc. (Cañadas, Luque \& Crespo, 2007).
Otro aspecto interesante era la concesión de cargos permanentes a personal especializado, como era el caso también de los boticarios, barberos, cirujanos y médicos (Valverde \& Vidal, 1975); o la recurrencia de forma eventual en el caso de las comadronas o parteras (García, García \& Valle, 1996).

Así mismo, otra parte fundamental dentro del documento era aquella en la que se habla de la remuneración de la labor asistencial. El enfermero de la reina Isabel de Valois tenía una ración diaria de cuatro panecillos, vino; y dos libras de carne; los días de pescado, se le daba la equivalencia en tal producto correspondiente a las dos libras de carne mencionadas. Además, obtenía una libra de merluza, de la cual dos sextas partes eran para el enfermero y el resto para uno de sus mozos, y podía obtener menudillos de las aves y las sobras de "las dolientes", a discreción del confitero que servía a la Corte. Finalmente, existen datos que permiten afirmar que percibía una quitación por su trabajo y de que se le debía abonar el dinero invertido en gastos para el servicio de las enfermas (Cañadas, Luque \& Crespo, 2007).

\section{DISCUSIÓN}

En el caso de los enfermeros y de los boticarios, encargados del mantenimiento y aprovisionamiento de la Enfermería y de la Botica Real, eran figuras destacadas dentro de la Corte, siendo escogidos al igual que por ejemplo los médicos (Valverde \& Vidal, 1975). Con respecto a las matronas, al no ejercer su práctica de forma habitual, sólo eran requeridas para los alumbramientos en la Corte, siendo seleccionadas en función de su experiencia y renombre; un aspecto curioso en este último caso, era que su atuendo pretendía ir acorde al status social de la mujer a la que asistiera en un momento dado (Valverde \& Vidal, 1975).

La aplicación de tratamientos múltiples lleva de forma inevitable a un control exhaustivo para que el personal sanitario conozca la posología administrada en cada momento. Destacable en la documentación es el hecho de que se anotasen en que momento del día y la forma de presentación del fármaco, lo que concuerda con la jornada laboral del personal de la época (Chocarro, Melgar \& Pérez, 1996; Eseverri, 1996); dicho control tam- 
bién se empleaba en el control de gastos de la Enfermería y de la Botica Real, permitiendo la gestión y la contabilidad de estas dependencias.

Las relaciones mencionadas anteriormente atienden a la necesidad de llevar una buena organización. El material médico y asistencial aparecía perfectamente catalogado según al paciente al que perteneciese, lo que se presume que se debía gestionar de forma racional los recursos materiales (Valverde \& Vidal, 1975). Además, se puede afirmar que, a pesar de ser un elenco de lo más básico y genérico en algunos casos, destaca la importancia que se le daba a la individualidad de los pacientes y de sus enseres personales, perfectamente identificados (Cañadas, Luque \& Crespo, 2007).

Las retribuciones del personal sanitario, además de la compensación económica en sí, estaba constituida por un sustento alimenticio acordado previamente. Exceptuando situaciones excepcionales, la quitación normal era de una cantidad económica acorde a la categoría, así como de una ración de carne de vaca o pescado, siendo este último el mas empleado (Cañadas, Luque \& Crespo, 2007).

\section{CONCLUSIONES}

El personal sanitario gozaba de la confianza del Rey y su círculo, lo cual queda sobradamente demostrado, dado el número de profesionales que atendían de forma constante las dolencias del ámbito regio. Tanto si era personal asiduo y permanente dentro de ella como si de forma eventual eran requeridos para prestar sus servicios (García, García \& Valle, 1996), el personal sanitario gozaba de una erudición y una profesionalidad metódi-

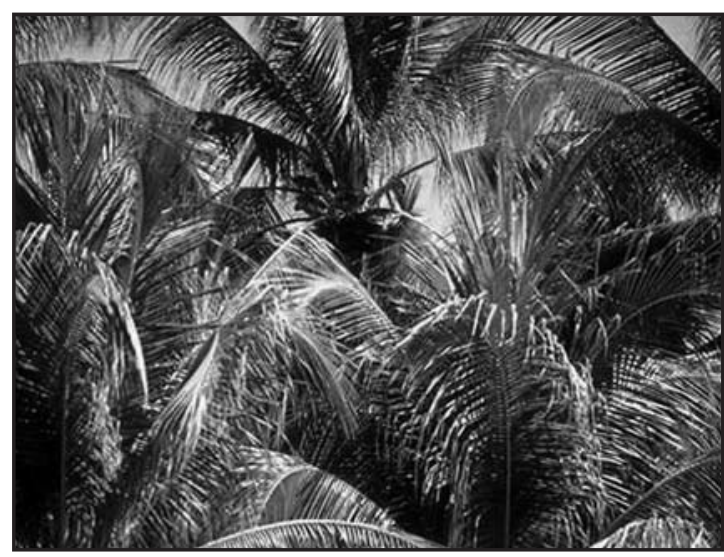

ca propias de su oficio (García Martínez, 2004, A; García Martínez, 2004, B), lo cual les confería un savoir fair con el que atendían, de forma notable, al personal cortesano. Todo ello obviamente se derivaba de la enseñanza, más o menos reglada, y la existencia de textos que apoyaban su formación (García, García \& Valle, 1996; García Martínez, 2004, A; García Martínez, 2004, B; Nogales, 2003), sin olvidar, claro está, que sería esa actitud metódica la que daría paso a la evolución del arte de cuidar, dándole un enfoque integrado, y la aplicación del método científico a éste (García Martínez, 2004, A; García Martínez, 2004, B; Nogales, 2003; Conde, 2002; García-Carpintero, 2007).

Queda patente la importancia que tenía recoger de forma exacta el gasto tanto del material y medicamentos para la enfermería, así como de lo que debía de percibir el personal encargado de la salud de la Corte (Cañadas, Luque \& Crespo, 2007; Valverde \& Vidal, 1975). En el caso de los tratamientos aplicados a Enrique IV, las diversas anotaciones permitían llevar un control bastante exhaustivo de los tratamientos que se realizaban, permitiendo así controlar su evolución y llevar un registro escrito de las actividades y posibles incidencias que se producían diariamente. Otro de los datos recavados y que resulta de interés es la figura destacada de D. Diego Ortega, sangrador real, cuyo papel como administrador, único al parecer, lo hacía responsable de los recursos tanto humanos como económicos de la enfermería, fundamentalmente porque las cuentas correspondientes se abonaban a posteriori. Así, por un lado, dentro de la estructura de la Corte Real, en lo que a su aspecto financiero se refiere, es preciso una constancia por escrito de todo aquello que sucedía en la salvaguarda de la salud del círculo próximo a los reyes; por otro lado, los inventarios del material registraban de forma precisa los gastos de lo que era necesario para mantener unas condiciones higiénicosanitarias adecuadas al entorno cortesano de la época, las cuales se traducen, en su mayoría, en medidas preventivas que evitasen males mayores (Cañadas, Luque \& Crespo, 2007; Chocarro, Melgar \& Pérez, 1996; Eseverri, 1996; Luque, Cañadas, Crespo \& Cantero, 2006; De la Torre, 1987). 
Las condiciones higiénico-sanitarias eran fundamentales dentro de las funciones del personal sanitario subalterno. En condiciones generales, la higiene, las medidas dietéticas y la atención individualizada se llevaban a rajatabla en cualquier hospital de la época como medida básica de los cuidados al enfermo y prevención ante posibles complicaciones para ellos e incluso el personal sanitario, por ello no es de extrañar que tales medidas se extremasen ante dolencias padecidas por la Casa Real (Cañadas, Luque \& Crespo, 2007; Valverde \& Vidal, 1975; De la Torre, 1987).

Por último, destacaremos el conflicto consecuente de los salarios administrados por la labor desempeñada. No era ningún secreto que los trabajadores de la corte eran objeto de irregularidades en sus estipendios, por lo que era habitual el reclamo por parte de los afectados de lo que les correspondía por derecho; es el caso de D. Antonio Martínez, enfermero de la Corte (Cañadas, Luque \& Crespo, 2007; Valverde \& Vidal, 1975) llama la atención la alusión al perjuicio que supone que se le paguen sus emolumentos en pescado y no en carne, justificándolo por su bajo coste y constatando así la creencia general que se tenía de considerar a la carne como alimento de primer orden, consideración de la que no gozaba el pescado, entre otros muchos alimentos (Reitz, 1991; Crespo, Cañadas, Luque \& Cantero, 2006).

\section{FUENTES DOCUMENTALES}

Archivo General de Simancas, Casa y Sitios Reales

1. Legajo 40, folio 4, 113; folio 21, 685-690; folio $36,941-942$.

2. Legajo 97, folios 1-55.

\section{BIBLIOGRAFÍA}

- Campos Díez, M.S. (2002). Las enfermerías de damas y criadas en la corte del siglo XVII. Dynamis, 22, 59-84.

- Cañadas de la Fuente, G.A., Crespo Muñoz, F.J. \& Luque Sánchez, M.E. (2003). Los inventarios como fuente para la historia de ciencias de la Salud. Boletín de la Sociedad Española de Ciencias y Técnicas Historiográficas, 3, 257-266. - Cañadas de la Fuente, G.A., Luque Sánchez, M.E. \& Crespo Muñoz, F.J. (2007). La asistencia sanitaria en la corte de Isabel de Valois. INDEX de Enfermería, 16 (58), 75-77.

- Chocarro, L., Melgar, G. \& Pérez, A. (1996). La Enfermería en las Ordenanzas y Constituciones del Hospital General y Agregados de Madrid. Año 1589. Híades, 3, 147-166.

- Conde Gutiérrez, F. (2002). Encuentros y desencuentros entre

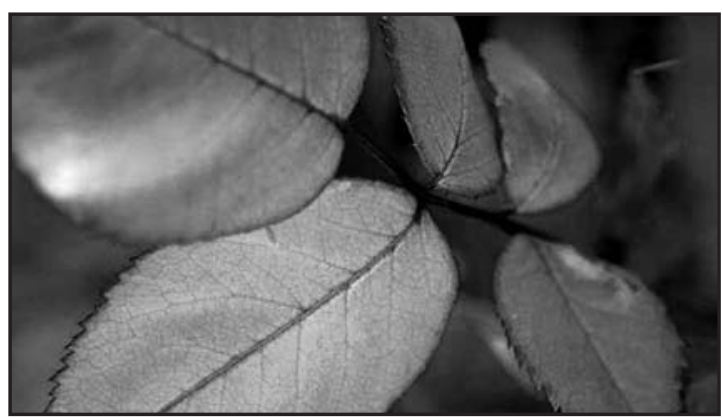

la perspectiva cualitativa y la cuantitativa en la historia de la medicina. Revista Española de Salud Publica, 76 (5), 395-408. Crespo Muñoz, F.J., Cañadas de la Fuente, G.A., Luque Sánchez, E. \& Cantero Hinojosa, J. (2006). La alimentación del siglo XVI: las evidencias documentales en el Archivo General de Simancas. En Universidad de Granada (ed.), Actas de las III Jornadas Científicas Nacionales y I Internacionales de Ciencias de la Salud (pp. 236-237). Granada: autor.

- De la Cuesta Benjumea, C. (2006). Naturaleza de la investigación cualitativa y su contribución a la práctica de Enfermería. Metas de enfermería, 9 (5), 50-55.

- De la Torre Díaz, J.L. (1987). El poder y la magia: la superstición en la corte de la España Moderna. Historia 16, 12 (136), 57-63.

- De los Santos Alfonso, J.M. (1999). Del cuidado natural a la Enfermería Profesional. Híades, 5-6, 75-91.

Eseverri, C (1997). La Enfermería medieval. Un documento histórico. Híades, 3, 229-254.

Eseverri, C. (1996). La Enfermería medieval. Un documento histórico. Híades, 3, 229-249.

- Fernández, A. (1617). Instrucción de enfermeros. Madrid: autor.

García Martínez, M.J. (1999). Las Carreras Auxiliares Médicas. Híades, 5-6, 55-60.

García Martínez, M.J. (2004, A). El método en la Enfermería: Una mirada desde la Historia (siglo XVII). Metas de Enfermería, 7 (10), 58-64.

García Martínez, MJ (2004, B). Cuidados enfermeros en la España del siglo XVII. Hacia la búsqueda de una identidad profesional. Gazeta de Antropología, (20).

- García, A.C., García, M.J. \& Valle, J.I. (1996). La imagen de la matrona en la Baja Edad Media. Híades, 3, 61-84.

García-Carpintero Blas, E. (2007). Reflexión del papel de la enfermería a lo largo de la historia. Enfermería global, 6 (11). Luque Sánchez, E., Cañadas de la Fuente, G.A., Crespo Muñoz, F.J. \& Cantero Hinojosa, J. (2006). Un aspecto interesante de la medicina de los siglos XV y XVI: recetas y conjuros. En Universidad de Granada (ed.), Actas de las III Jornadas Científicas Nacionales y I Internacionales de Ciencias de la Salud (pp. 238-239). Granada: autor.

Nogales Espert, A. (2003). Evolución histórica del método enfermero. Cultura de los cuidados, 7 (13), 23-28.

- Reitz, J.E. (1991). Dieta y alimentación hispano-americana en el Caribe y la Florida en el siglo XVI. Revista de Indias, 51, 11-24.

- Valverde, J.L. \& Vidal, M.C. (1975). Cuentas de la Real Botica desde 1571 a 1695, según documentación del Archivo del Palacio Real de Madrid. Ars Pharmaceutica, 16, 393-364. 